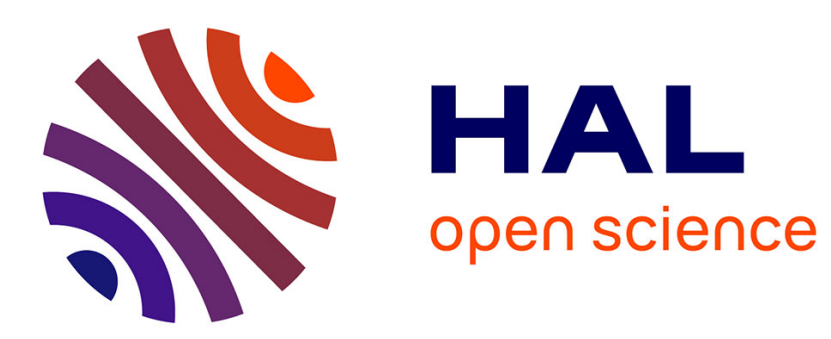

\title{
Dysfonctionnements du système de récompense et biais cognitifs chez les joueurs pathologiques
}

\author{
Jean-Claude Dreher
}

\section{To cite this version:}

Jean-Claude Dreher. Dysfonctionnements du système de récompense et biais cognitifs chez les joueurs pathologiques. Bulletin de l'Académie Nationale de Médecine, 2021, 205 (7), pp.748-760. 10.1016/j.banm.2021.05.010 . hal-03369337

\section{HAL Id: hal-03369337 \\ https://hal.science/hal-03369337}

Submitted on 7 Oct 2021

HAL is a multi-disciplinary open access archive for the deposit and dissemination of scientific research documents, whether they are published or not. The documents may come from teaching and research institutions in France or abroad, or from public or private research centers.
L'archive ouverte pluridisciplinaire HAL, est destinée au dépôt et à la diffusion de documents scientifiques de niveau recherche, publiés ou non, émanant des établissements d'enseignement et de recherche français ou étrangers, des laboratoires publics ou privés. 


\section{Proof Central}

\section{Dear Author}

Please use this PDF proof to check the layout of your article. If you would like any changes to be made to the layout, you can leave instructions in the online proofing interface. First, return to the online proofing interface by clicking "Edit" at the top page, then insert a Comment in the relevant location. Making your changes directly in the online proofing interface is the quickest, easiest way to correct and submit your proof.

Please note that changes made to the article in the online proofing interface will be added to the article before publication, but are not reflected in this PDF proof.

If you would prefer to submit your corrections by annotating the PDF proof, please download and submit an annotatable PDF proof by clicking the link below.

\section{$[$ Annotate PDF}




\section{Dysfonctionnements du système de récompense et biais cognitifs chez les joueurs pathologiques}

\section{Reward system dysfunctions and cognitive biases among pathological gamblers}

\section{\& Q1 Jean-Claude Dreher}

Neuroéconomie, récompense et prise de décision, Centre de neurosciences cognitives, CNRS, Institut des

Sciences Cognitives Marc Jeannerod, 67, boulevard Pinel, 69675 Bron cedex, France

Reçu le 26 mars 2021 ; accepté le 26 avril 2021

\section{MOTS CLÉS \\ Addiction ; \\ Joueurs ; \\ Mécanismes ; \\ Cerveau}

\section{KEYWORDS \\ Addiction; \\ Gamblers; \\ Mechanism; \\ Brain}

\begin{abstract}
Résumé Les addictions comportementales incluent l'addiction aux jeux de hasard et d'argent ou jeu pathologique, addiction aux jeux vidéo, achats compulsifs, addictions alimentaires, dépendance à l'activité physique, dépendances sexuelles et affectives, etc. Il existe des similitudes neurobiologiques, psychopathologiques et comportementales entre les addictions avec produit et les addictions comportementales. Le but de cette présentation est d'illustrer de façon simple les changements cérébraux que nous avons observés dans le jeu pathologique grâce à la neuro-imagerie (IRM fonctionnelle) lors d'expériences sollicitant le système de récompense ou des biais cognitifs. Les résultats permettent de mieux comprendre l'engagement des régions cérébrales et les mécanismes mis en jeu lors du traitement de récompenses et de décisions inadaptées dans le jeu pathologique.
\end{abstract}

๑ 2021 l'Académie nationale de médecine. Publié par Elsevier Masson SAS. Tous droits réservés.

Summary Behavioral addiction include addiction to gamble, video game addiction, compulsive shopping, food addiction, physical activity addiction, sexual and emotional addiction... There are neurobiological, psychopathological and behavioral similarities between addiction with product and behavioral addiction. The aim of this presentation is to illustrate in a simple way the brain changes observed by our team in pathological gamblers using neuroimaging (functional MRI) during paradigms engaging the reward system and cognitive biases. The results provide

\footnotetext{
Séance du 2 mars 2021.

Adresse e-mail : dreher@isc.cnrs.fr

1 Pour un accès direct aux publications citées voir lien ci-dessous :

https://dreherteam. wixsite.com/neuroeconomics/publications.
} 
a better understanding of brain regions and mechanisms involved in reward processing and decision making in pathological gambling.

๑ 2021 l'Académie nationale de médecine. Published by Elsevier Masson SAS. All rights reserved.

\section{Introduction ${ }^{1}$}

La dépendance à un comportement est caractérisée par l'impossibilité répétée de résister à la répétition de ce comportement. Les aspects cliniques en sont : sensation de tension croissante précédant l'amorce du comportement, plaisir (ou soulagement) ressenti durant la réalisation du comportement avec sensation de perte de contrôle.

Les addictions comportementales peuvent se définir comme le résultat d'une interaction entre un individu et un objet externe banal utilisé par tous : les jeux, les achats, la nourriture, le sport... Elles incluent l'addiction aux jeux de hasard et d'argent ou au jeu pathologique, l'addiction aux jeux vidéo, les achats compulsifs, les addictions alimentaires, la dépendance à l'activité physique et les dépendances sexuelles.

\section{Qui est concerné par les addictions comportementales?}

Les enquêtes épidémiologiques sont peu nombreuses. Quelques estimations cependant :

- entre 5,8 et $7 \%$ de la population présenterait une addiction aux achats :

- entre 0,1 et 5-8 \% serait dépendante des jeux de hasard et d'argent ;

- entre 1 et $4 \%$ serait anorexique et 1 à $2 \%$ boulimique ;

- environ $3 \%$ développerait une addiction au sport ;

- et entre 2 et $6 \%$ au sexe...

Parmi les facteurs de vulnérabilité on trouve :

- les facteurs liés à l'objet même de l'addiction ;

- les troubles de l'humeur ;

- les troubles anxieux ;

- les troubles de personnalité ;

- des facteurs d'environnement :stress, contexte social, familial....

La pratique des jeux de hasard et d'argent est de plus en plus diverse, au point aujourd'hui d'être partie intégrante de la vie quotidienne.

Trois conditions définissent les jeux de hasard et d'argent : le joueur mise de l'argent ou un objet de valeur ; une fois placée cette mise ne peut être reprise ; l'issue du jeu repose sur le hasard. Bien que ce type de jeux constitue chez la plupart des personnes un passe-temps ludique, d'autres vont développer des comportements compulsifs. On identifie ainsi généralement trois types de joueurs, qui représentent trois types de comportements associés au jeu.

Le premier type est le joueur « récréatif » pour lequel le jeu n'est pas un problème. Le deuxième type est le joueur «problématique » qui contrôle mal son comportement bien qu'il en subisse des aspects négatifs. Le troisième type est le joueur «pathologique » qui fait face à une dépendance aux jeux de hasard et d'argent. La prévalence de ce dernier type de comportement est estimée entre 1 et $2 \%$ dans la plupart des pays, avec des chiffres très variables d'une étude à l'autre.

Depuis son intégration dans le Manuel Diagnostique et Statistique des troubles mentaux de l'association américaine de psychiatrie (DSM III), le jeu pathologique a suscité l'intérêt des chercheurs, mais reste encore peu étudié, surtout en France. La recherche s'est surtout concentrée sur des aspects cliniques, à travers des études épidémiologiques, le développement d'échelles diagnostiques, des stratégies de prévention, les traitements. Beaucoup moins de travaux à visée fondamentale ont été réalisés, mis à part quelques études qui se sont intéressées aux facteurs de risque sur le plan génétique ainsi qu'aux substrats neurobiologiques. La recherche dite fondamentale en est à ses débuts : il est très important aujourd'hui de fournir un effort afin de mieux comprendre l'ensemble des facteurs influençant le développement, le maintien voire d'identifier des pistes de traitement.

Ces dernières années a émergé un début de modèle explicatif : en effet, tout comme l'achat compulsif, le jeu pathologique est souvent considéré comme une addiction sans produit psychoactif, c'est à dire une " addiction comportementale ». Selon le DSM-IV, le jeu pathologique, qui se trouve dans la catégorie des «troubles du contrôle des impulsions non classés dans les autres catégories », est défini comme une «pratique inadaptée, persistante et répétée du jeu ». Le jeu pathologique fournit un modèle heuristique d'addiction sans drogue, où les marqueurs biologiques ne peuvent être expliqués par les effets d'une molécule ayant généré un état de dépendance. Cependant au sein même de ce modèle plusieurs hypothèses s'affrontent pour expliquer la persévération des joueurs pathologiques dans leur comportement : dysfonctionnement des processus de prise de décision ? Sur-sensibilité aux gains ou une sous-sensibilité aux pertes?

Notre équipe étudie la prise de décision et le système de récompense chez l'homme sain et dans diverses maladies neurologiques ou psychiatriques à l'aide d'une approche de neuroéconomie qui combine l'imagerie par résonance magnétique fonctionnelle (IRMf) et l'économie comportementale, science dédiée à l'étude des comportements qui caractérisent l'être humain dans des situations de choix économiques [1]. C'est avec cette approche que nous étudions également les bases neurobiologiques du jeu pathologique. Cette piste a encore été très peu explorée.

\section{Les biais cognitifs sont à l'origine de comportements inadaptés des joueurs pathologiques}

Les biais cognitifs liés au jeu sont des pensées erronées, des croyances dysfonctionnelles, des erreurs d'appréciation 

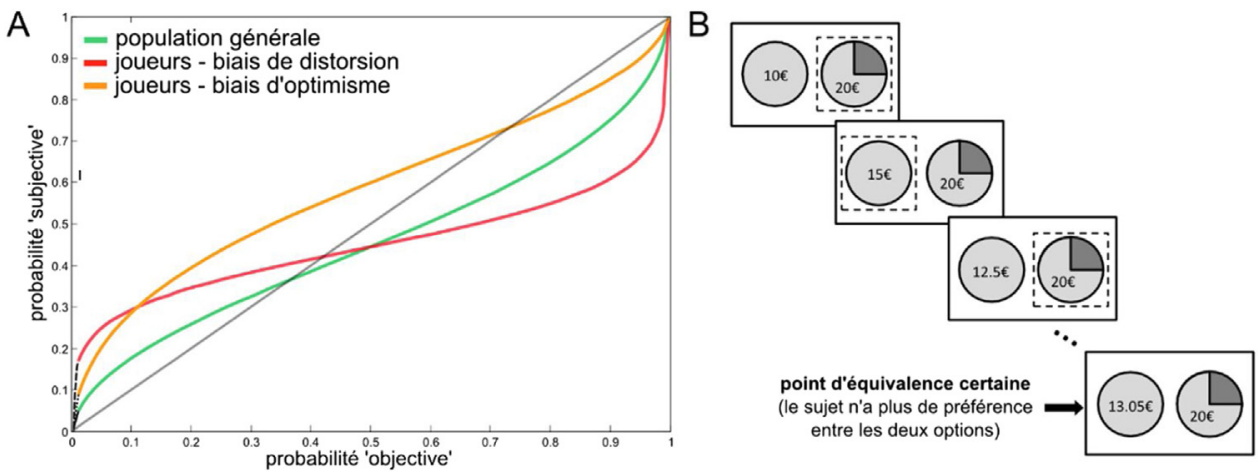

Figure 1 Le graphique de gauche présente le comportement typique observé dans la population générale en vert, qui se caractérise par une courbe en 'S-inversé' révélatrice d'une distorsion des probabilités. L'hypothèse de distorsion exagérée chez les joueurs, telle que dérivée des travaux de Kahneman et Tversky est représentée en rouge tandis que l'hypothèse alternative dite d'optimisme est quant à elle représentée en orange. Un individu parfaitement rationnel verrait sa courbe adhérer à la diagonale grise. Le schéma de droite décrit la procédure utilisée : le sujet doit effectuer une série de choix entre des montants surs et des paris risqués (ici, $20 €$ avec $75 \%$ de chances de gagner et des montants certains variables), les décisions proposées étant ajustées jusqu’à ce que le sujet soit indifférent entre les deux options (point d'équivalence certaine, ici $=13,05 €$ ).

ou de jugement liées au facteur « hasard » de la pratique des jeux de hasard et d'argent. Ces biais apparaissent comme un des facteurs de risque impliqué dans l'initiation et le maintien d'une pratique pathologique du jeu. Ces biais cognitifs sont partagés par tous les joueurs, mais ils permettent de différencier joueurs non pathologiques et joueurs pathologiques, car en situation de jeu ces derniers expriment davantage de pensées erronées. Ces biais pourraient favoriser la poursuite ou le retour au jeu afin de récupérer l'argent perdu.

Un biais cognitif très fréquent dans la population générale est la distorsion des probabilités qui apparaît lorsque l'on demande aux gens de choisir entre un gain d'argent sûr et un gain d'argent plus important mais dont la probabilité de survenir est inférieure à $100 \%$. Répétés plusieurs fois au sein d'un protocole standard qualifié d'équivalence certaine, ce type de choix permet de montrer que la plupart d'entre nous a tendance à surévaluer les petites probabilités de gains et à sous-évaluer les grandes probabilités de gains. Autrement dit, du point de vue cognitif, les êtres humains ont une sensibilité irrégulière aux probabilités de gains qui se traduit par une irrationalité dans leurs décisions économiques. C'est au début des années 70, que Kahneman et Tversky [2] ont entamé une série de travaux portant sur la prise de décision en situation d'incertitude qui devait les amener à recevoir le prix Nobel d'économie en 2002. Leur célèbre «théorie des perspectives » [3] constitue un des fondements l'économie comportementale. Le travail central de ces chercheurs porte sur la manière dont nous nous comportons lorsqu'il s'agit de prendre des risques financiers. Indépendamment des nombreuses conséquences de cette découverte pour le monde de la finance et des assurances, Kahneman et Tversky ont proposé que le biais de distorsion pourrait jouer un rôle dans l'attraction des êtres humains pour les jeux d'argent, qui impliquent le plus souvent de petites probabilités de gagner.

Dès lors, l'hypothèse d'un biais de distorsion exagéré devenait une hypothèse plausible pour expliquer le penchant pathologique de certains individus vis-à-vis des jeux d'argent. Cette idée n'avait pourtant jamais été testée : les joueurs compulsifs sont-ils victimes de ce biais cognitif que nous partageons tous ou bien entretiennent-ils avec les probabilités un rapport spécifique et caractéristique de leur pathologie?

Nous avons cherché à répondre à cette question en utilisant la tâche d'équivalence certaine (décisions répétées entre des montants certains et des montant incertains) chez des joueurs et chez des sujets sains appariés en âge, niveau d'études, consommation d'alcool, de cigarettes, etc [4] (Fig. 1).

À partir des résultats de cette expérience, nous avons pu tout d'abord reconstruire la représentation des probabilités de chaque sujet. Ensuite, une procédure mathématique simple a permis de quantifier chez chaque participant deux paramètres régissant la forme de cette représentation : le premier renvoyant directement à l'intensité du biais de distorsion, le second correspondant à la surestimation (ou la sous-estimation) générale de l'ensemble des probabilités. Le résultat de ces analyses fut doublement surprenant : non seulement le biais de distorsion était très similaire entre les joueurs pathologiques et les sujets contrôles, mais il est apparu que le second paramètre associé à la surestimation générale des probabilités était très significativement différent entre les deux groupes [4] (Fig. 2).

Autrement dit, les joueurs pathologiques se démarquaient largement des individus normaux en présentant un biais cognitif inattendu dans le traitement des probabilités de gains monétaires : quelle que soit la probabilité considérée, ces sujets ont tendance à surestimer la valeur des options risquées. Encore plus frappant, l'intensité de cet effet était corrélée à la sévérité des symptômes. Cette découverte signifie que les joueurs pathologiques ne souffrent pas d'une sensibilité accrue (i.e. distorsion exagérée) aux faibles probabilités de gains, comme suggéré par les travaux de Kahneman et Tversky, mais qu'ils ont un biais d'optimisme reflétant une préférence systématique pour les situations qui impliquent une prise de risque financière, et cela quelle que soit la probabilité de gains monétaires [4]. La simplicité de la procédure utilisée pour aboutir à ce résultat ainsi que la fiabilité des mesures 


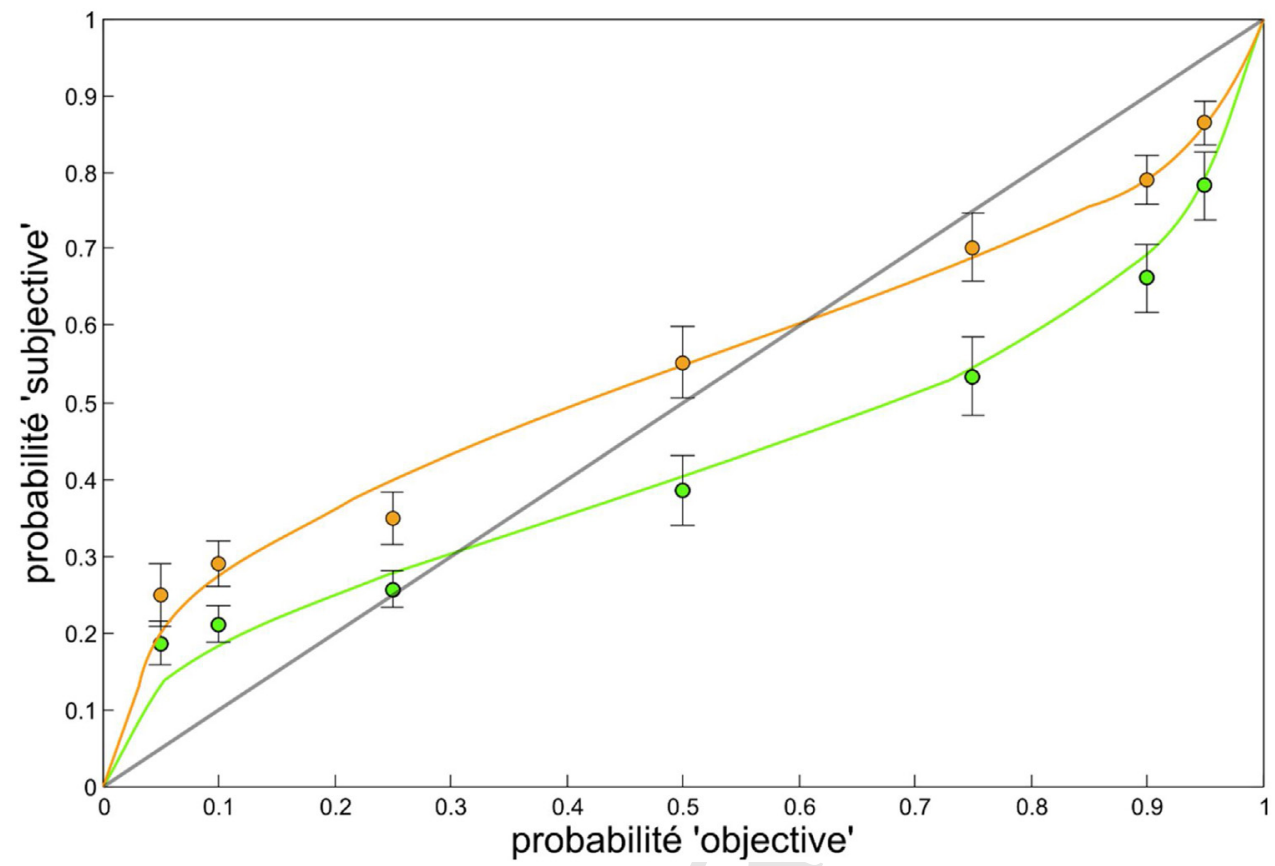

Figure 2 Les deux courbes représentées décrivent le comportement du groupe de sujets contrôles (en vert) et le groupe de joueurs pathologiques (en orange). À l'examen de ce graphique, il est évident que les joueurs surestiment l'ensemble des probabilités comparativement aux contrôles et l'on remarque aussi que la distorsion de cette courbe est tout-à-fait similaire entre les deux groupes.

pourraient permettre d'exporter ce test vers la pratique clinique à deux fins distinctes : tout d'abord, en tant qu'outil de diagnostic supplémentaire pour les psychiatres qui s'intéresseraient au maniement des probabilités de gains par leurs patients, et ensuite, comme moyen de faire prendre conscience à ces mêmes patients de l'existence d'un biais cognitif qui a un impact direct sur leur manière d'appréhender les situations à risque.

Les causes neurobiologiques de ce biais, qualifié d'optimiste, sont encore inconnues à l'heure actuelle mais nous avons une étude de neuro-imagerie en cours pour répondre à cette question. Malgré tout, du point de vue neurocognitif, un tel biais peut avoir plusieurs origines qui ramènent toutes à l'idée de sensibilité aux gains et aux pertes d'ordre financier ayant un impact direct sur le plaisir et le déplaisir, attendu ou éprouvé par les joueurs lorsque des sommes d'argent réelles sont en jeu. L'hypothèse d'une moindre sensibilité aux pertes monétaires, potentialisant par contraste la valeur attendue de toute option risquée est fondée sur l'idée du déséquilibre d'un système, dit de « récompense »dans le jeu pathologique. Une première approche fondamentale est donc d'étudier le rôle des composantes de ce système chez l'homme sain, avant de chercher le dysfonctionnement propre aux joueurs pathologiques.

\section{Études de neuro-imagerie sur le système de récompense}

Les structures cérébrales qui composent le système de récompense ont en commun d'être innervées principalement par les neurones dopaminergiques des voies mésocorticolimbique et nigro-striée, prenant naissance respectivement dans l'aire tegmentale ventrale et la substance noire du mésencéphale (Fig. 3).

La dopamine libérée au niveau des sites de projection des neurones dopaminergiques vient moduler un grand nombre de processus cognitifs, comme l'apprentissage, la motivation et les émotions. Cependant ces fonctions sont très générales, et pour comprendre le mode de fonctionnement du système de récompense, il est fondamental de comprendre quels signaux théoriques les neurones dopaminergiques codent. Ainsi chez le primate non humain, l'enregistrement direct des neurones dopaminergiques par l'équipe de Wolfram Schultz à Cambridge a permis de montrer que leurs décharges correspondent à trois mesures théoriques qui sont

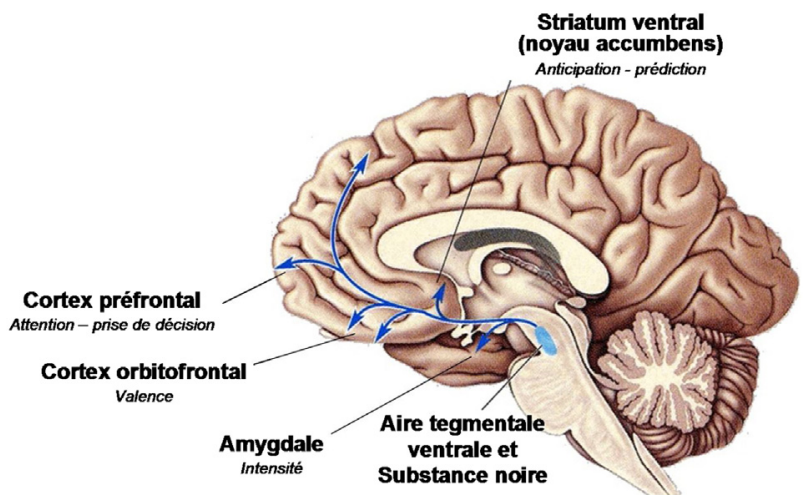

Figure 3 Représentation schématique des sites de projection de la voie dopaminergique mésocorticolimbique impliqués dans le système de récompense. Le rôle principal attribué à chaque structure au sein de ce système est indiqué en italique. 
révélées à des moments précis du comportement [5]. Le protocole utilisé dans ces études est après un apprentissage conditionné de type pavlovien pendant lequel l'animal associe un stimulus avec une récompense probabiliste. Le premier signal codé par les neurones dopaminergiques correspond à la valeur subjective de la récompense (l'espérance mathématique est liée à son intensité multipliée par la probabilité des récompenses) qui apparaît de façon transitoire au moment du stimulus conditionné. Un second signal apparaît pendant la phase d'anticipation entre le stimulus conditionné et la récompense et coderait l'incertitude de la récompense (étant maximale quand l'animal a une chance sur deux d'être récompensé). Enfin, le troisième type de signal refléterait la différence entre la récompense attendue et la récompense délivrée, nommée l'incertitude de récompense des joueurs pathologiques.

L'utilisation de l'IRMf chez l'homme sain et d'un paradigme expérimental fondé sur celui élaboré chez l'animal a permis d'identifier les réseaux cérébraux qui représentent différents aspects liés à la probabilité des récompenses monétaires [6-9] (Fig. 4).

Dans cette expérience, les personnes à l'intérieur du scanner jouaient avec des «machines à sous » projetées sur un écran. Contrairement aux machines à sous d'un casino dont les chances de gagner sont inconnues, ici les probabili-

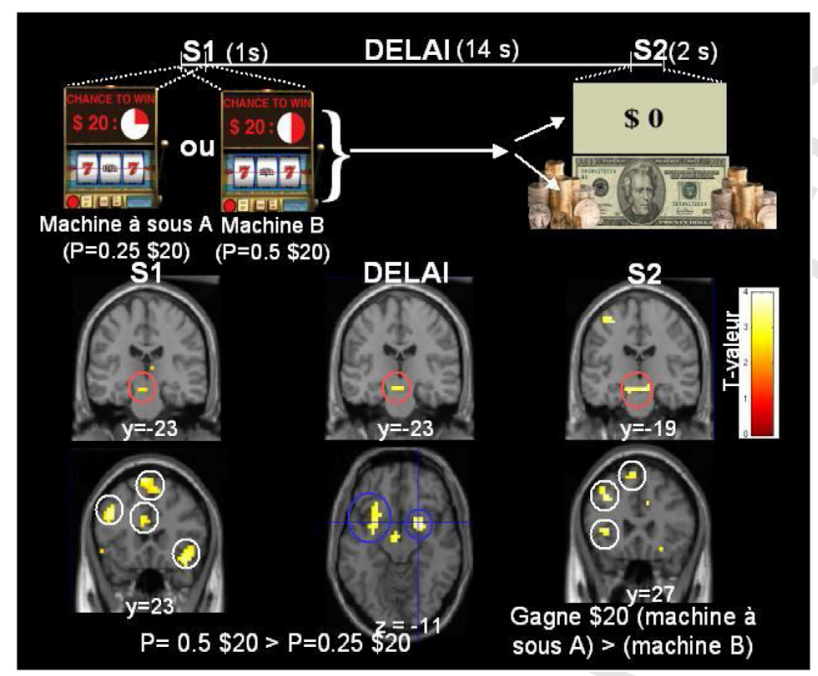

Figure 4 Dans une expérience d'imagerie par résonance magnétique cérébrale dans laquelle des sujets sains « jouaient » dans le scanner avec différentes machines à sous virtuelles présentant des probabilités de gains différents (et gagnaient effectivement de l'argent après le scanner). Les résultats ont montré que les régions contenant les neurones dopaminergiques répondent de manière transitoire au moment du stimulus prédisant la récompense S1 ainsi qu'au moment de l'apparition de la récompense $S 2$ et répondent de manière soutenue pendant la phase d'anticipation d'une récompense d'incertitude maximale (activité cerclée en rouge). De plus, le cortex préfrontal répond au signal transitoire (activité cerclée en blanc) tandis que l'activité du striatum ventral varie avec le signal soutenu (activité cerclée en bleue). Ces résultats indiquent que des réseaux neuronaux distincts traitent différents aspects des propriétés statistiques de l'information liées aux récompenses chez l'homme sain. tés de gagner avec les différentes machines étaient données de façon explicite. Ceci permettait d'étudier les réseaux cérébraux engagés par la représentation des probabilités subjectives de recevoir une récompense, de l'incertitude des récompenses et du signal de prédiction d'erreur des récompenses. Ces signaux sont présents après apprentissage dans les études d'enregistrement des neurones dopaminergiques chez l'animal et c'est pour cette raison que dans cette étude réalisée chez l'homme les probabilités de gagner étaient données de façon explicite. Il était ainsi possible de visualiser et de distinguer les régions cérébrales codant la valeur subjective de la récompense à venir au moment de la présentation de la machine, celles répondant en anticipation du gain incertain et celles répondant à la différence entre le gain attendu et celui effectivement reçu. Pris dans leur ensemble, les résultats de cette étude permettent de circonscrire le rôle fonctionnel des réseaux neuronaux traitant différents aspects des propriétés statistiques de l'information de la récompense.

Brièvement, deux régions principales émergent de nombreuses études d'IRMf : la région ventromédiale du cortex préfrontal qui code la valeur subjective des récompenses à la fois en anticipation et au moment de leur évaluation tandis que le striatum ventral code l'erreur de prédiction de la récompense et l'incertitude de la récompense pendant la période d'anticipation entre le stimulus prédicteur de la récompense et cette dernière. Mais le système de récompense n'est pas seulement impliqué dans l'anticipation et la réception de récompenses monétaires : il répond également pour des de récompenses dites primaires, qui sont innées comme la nourriture, ou le sexe. La plupart des études d'imagerie publiées chez l'homme utilisent l'argent comme incentif alors que chez l'animal la récompense est la nourriture (jus de fruit chez le singe). Une originalité de nos recherches de ces dernières années a été de comparer la représentation cérébrale des récompenses primaires (jus de fruit ou images érotiques délivrés dans le scanner), et celle des récompenses secondaires comme l'argent, acquise par apprentissage. Il a ainsi été montré que le cortex préfrontal ventromédian est également engagé en anticipation de récompenses et de punitions de différentes nature (primaire, secondaire) et de différents types, au moment de la réception des récompenses, et qu'il effectue aussi une comparaison au moment du choix entre les options disponibles $[6,10,11]$.

Cependant, ces régions cérébrales ne sont pas les seules à représenter les différents signaux théoriques répertoriés ci-dessus. Au cours de l'apprentissage, c'est-à-dire lorsque la probabilité d'être récompensé doit être apprise, d'autres structures participent au codage de l'incertitude, comme le cortex orbitofrontal ou l'hippocampe [12,13]. Ceci a été mis en évidence par des enregistrements intracrâniens chez des patients épileptiques pharmaco-résistants pour lesquels on a implanté des électrodes à des fins de résection des foyers épileptiques. Dans l'hippocampe, le signal d'incertitude n'apparaît pas uniquement pendant la période d'anticipation entre le stimulus annonçant la récompense et cette dernière, mais aussi au moment où la récompense est délivrée. Au moment de la réception de la récompense, ce signal pourrait indiquer le degré d'incertitude entre stimulus et récompense pour renforcer cette association si l'incertitude est forte. 
Une limitation intrinsèque à l'IRMf est qu'utilisée seule, cette technique ne permet pas d'identifier la nature des neuromodulateurs mis en jeu, comme la dopamine qui est si importante dans les processus d'apprentissage et les processus motivationnels. Nous avons surmonté cette difficulté en mettant en œuvre une approche de neuro-imagerie multimodale combinant l'IRMf à la tomographie par émission de positrons (TEP). Cela a permis de lier l'activation cérébrale en IRMf à une mesure indirecte de la synthèse de dopamine en TEP. Il a ainsi été montré lorsque des sujets réalisent la même tâche de «machines à sous » que le cortex préfrontal latéral est davantage engagé en anticipation et au moment de la réception de la récompense quand la synthèse de dopamine augmente chez des sujets jeunes et sains [14]. Une relation inverse était apparente chez les sujets âgés, ce qui permet à la fois de lier l'activation cérébrale observée en IRMf reflétant la phase de motivation pour l'obtention de la récompense et la phase hédonique à la synthèse de dopamine, et de montrer que cette relation s'inverse avec le vieillissement normal.

La tâche montrant un biais d'optimisme dans le jeu pathologique a aussi été utilisée pour tester l'hypothèse que la dopamine module la pondération de probabilité subjective, en examinant l'effet d'un médicament dopaminergique sur les comportements à risque, tant chez les individus sains que chez les joueurs pathologiques [15]. Le blocage sélectif des récepteurs dopaminergiques D2/D3 diminue la distorsion des probabilités gagnantes en rendant les participants plus linéaires dans leur estimation subjective des probabilités, et donc plus rationnels dans leur comportement décisionnel.

\section{Dopamine et vulnérabilité génétique aux addictions}

L'analogie entre la dépendance aux drogues et le jeu pathologique permet de mieux comprendre le substrat neurobiologique du jeu pathologique. La dépendance aux drogues implique un dysfonctionnement du système dopaminergique et certaines personnes atteintes de la maladie de Parkinson peuvent devenir des joueurs pathologiques suite à l'introduction d'un agoniste de la dopamine, ce qui confirme la piste dopaminergique. Il existe cependant encore peu de données cohérentes sur le dysfonctionnement dopaminergique précis chez les joueurs pathologiques et sur la vulnérabilité génétique à développer cette pathologie. Il est en fait difficile d'établir des relations causales entre des variations génétiques et le risque de souffrir de maladies psychiatriques complexes.

Plutôt que de chercher les gènes responsables du jeu pathologique, une autre approche consiste à chercher les associations entre certains gènes impliqués dans la transmission dopaminergique et des phénotypes intermédiaires, comme l'impulsivité, une dimension de la personnalité ou à déterminer l'effet de ces gènes sur la réponse du système de récompense. Ainsi, l'équipe de Trevor Robbins à Cambridge [16] a montré chez le rongeur que l'impulsivité est associée à de faibles concentrations de certains récepteurs dopaminergiques (D2, D3) dans le striatum ventral, et qu'elle est un bon marqueur comportemental de la propension à s'administrer des drogues addictives. Une approche complé- mentaire consiste à caractériser les réseaux cérébraux reflétant cette impulsivité chez les joueurs pathologiques et chez les sujets sains. Au niveau comportemental, un paradigme pour tester l'impulsivité est celui de la dévaluation de la récompense par un délai d'attente. Dans ce dernier cas, les sujets doivent choisir entre deux options dont la récompense est variable et plus ou moins différée dans le temps (par exemple choisir entre 20 euros maintenant et 40 euros dans une semaine). Les récompenses futures sont ainsi dévaluées avec l'augmentation du délai. La valeur attribuée à une option dépend donc du délai d'attente pour obtenir la récompense. Cette dévaluation par le délai permet de caractériser l'impulsivité : une personne qui dévalue fortement les récompenses différées sera considérée comme plus impulsive qu'une personne capable d'attendre plus longtemps pour une meilleure récompense. Les joueurs pathologiques, comme d'autres patients souffrant d'addictions aux drogues, ont une plus forte impulsivité que des sujets sains. Au niveau cérébral, ces joueurs révèlent également une augmentation de la réponse du système de récompense avec la valeur subjective de la récompense menant à l'option différée dans le temps, comme l'a montré l'équipe de Christian Büchel à Hambourg [17]. Ces résultats ont initialement été interprétés par l'atténuation de la réponse du système de récompense chez les joueurs pathologiques. Nous avons par ailleurs montré qu'il est possible d'étudier la représentation cérébrale de cette impulsivité en utilisant des délais réellement éprouvés dans le scanner à l'aide de récompenses primaires : ce sont les mêmes réseaux cérébraux qui sont engagés tant lors de la dévaluation d'une image érotique que dans le cas d'une récompense monétaire chez des sujets jeunes [18].

Cette même expérience testant l'impulsivité pour des récompenses primaires avec des délais réels a aussi été utilisé pour étudier les troubles du contrôle des impulsions de patients Parkinsoniens [19]. Ces troubles sont liés à la maladie et/ou aux traitements dopaminergiques et altèrent la qualité de vie des patients parkinsoniens au même titre que les symptômes moteurs. Dans une étude récente, nous avons identifié les mécanismes neurophysiopathologiques sous-tendant les dérégulations de la prise de décision observées chez des patients ayant un trouble du contrôle des impulsions bien spécifique : l'hypersexualité.

Les patients atteints de la maladie de Parkinson (MP) peuvent développer des troubles du contrôle des impulsions ( $\mathrm{TCl}$ ) sous traitement dopaminergique. Les $\mathrm{TCl}$ englobent un large éventail de comportements, tels que l'hypersexualité, le jeu pathologique ou les achats compulsifs. Cependant, les systèmes cérébraux engagés dans des $\mathrm{TCl}$ spécifiques restent mal caractérisés.

Dans cette nouvelle étude utilisant l'imagerie par résonance magnétique fonctionnelle (IRMf), les chercheurs ont cherché à déterminer quels systèmes cérébraux sont engagés dans l'attente d'images érotiques chez des patients atteints de MP ayant une hypersexualité $(M P+H S)$, chez des patients sans hypersexualité (MP-HS) et des participants témoins non-atteints. Les patients atteints de MP ont été évalués avec ou sans traitement lévodopa (L-Dopa). Placés dans l'IRMf, les participants devaient choisir entre deux options : attendre 1,5 seconde pour visualiser brièvement une image érotique, ou attendre plus longtemps pour voir l'image érotique plus longtemps. Au moment de la prise 

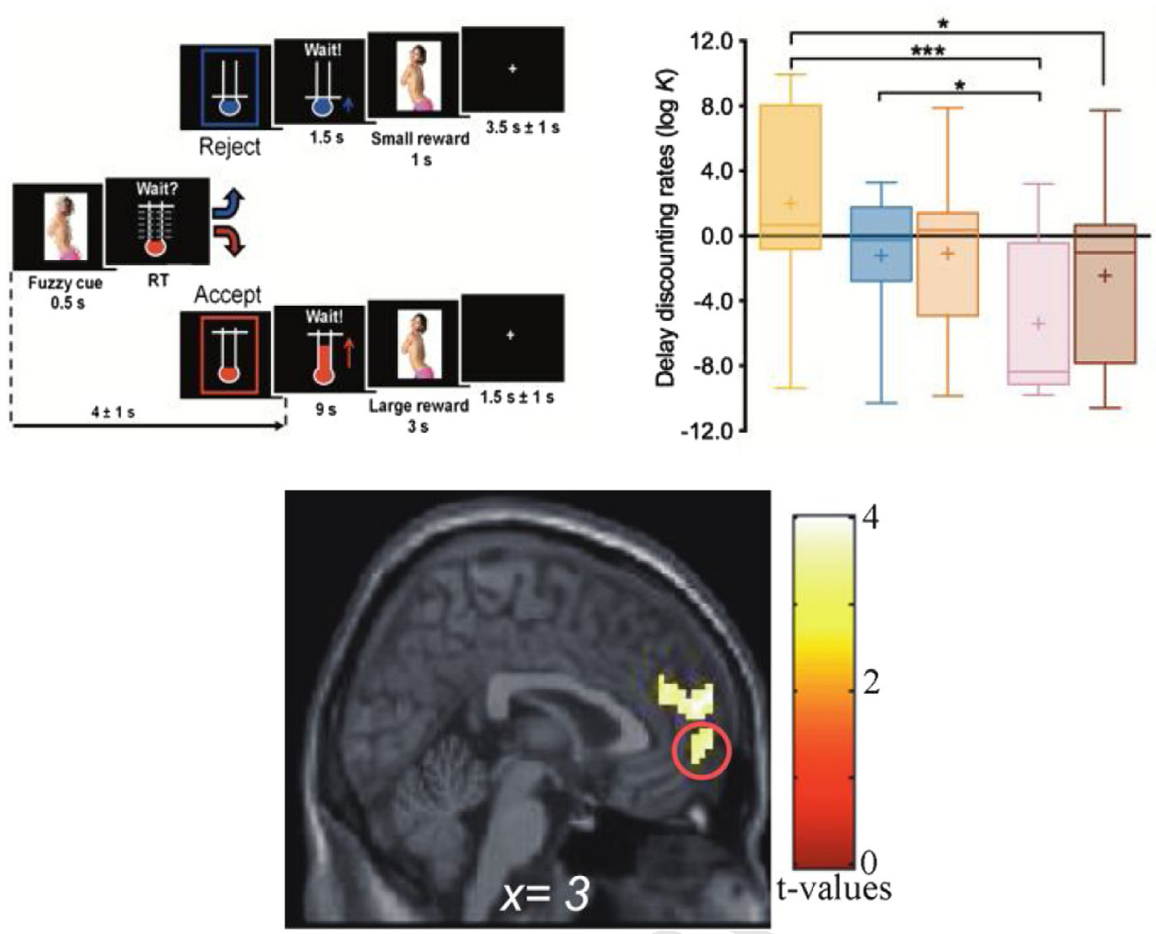

Figure 5 En haut à gauche. Expérience d'IRMf réalisée chez des patients Parkinsoniens souffrant d'une addiction comportementale spécifique : l'hypersexualité. Placés dans le scanner, les participants devaient choisir entre deux options : attendre 1,5 seconde pour visualiser brièvement une image érotique, ou attendre plus longtemps pour voir l'image érotique plus longtemps. Au moment de la prise de décision, l'équipe a identifié quelles régions du cerveau étaient impliquées dans l'évaluation de ces deux options. À droite. Les patients MP + HS étaient prêt à attendre plus longtemps (taux de décompte temporel moins important) les images érotiques différées que les patients MP-HS et les témoins. (PD + HS : Parkinsoniens ayant des troubles de l'Hypersexualité. PD-HS : Parkinsoniens sans Hypersexualité. On : traitement L-DOPA ; OFF : sans traitement). En bas. Au niveau cérébral, les PD + HS, comparés aux contrôles et à PD-HS, engageaient d'avantage le cortex préfronto-ventromédial, lorsque la durée de l'attente proposée augmentait. Cette région cérébrale est connue pour évaluer les différentes options proposées pour présider à la décision.

de décision, l'équipe a identifié quelles régions du cerveau étaient impliquées dans l'évaluation de ces deux options.

Les patients $(\mathrm{MP}+\mathrm{HS})$ étaient prêts à attendre plus longtemps les « récompenses » (images érotiques) différées que les patients (MP-HS) et les témoins. Ainsi, en utilisant des stimuli érotiques qui les motivent, les patients $(M P+H S)$ se montraient en fait moins impulsifs pour la récompense immédiate. Au niveau cérébral, les ( $M P+H S)$, comparés aux contrôles et aux (MP-HS), engageaient d'avantage le cortex préfronto-ventromédial, lorsque la durée de l'attente proposée augmentait, alors que l'inverse était vrai pour les groupes contrôles. Cette région cérébrale est connue pour évaluer les différentes options proposées pour présider à la décision. De plus, chez les patients $(M P+H S)$, le traitement à la L-Dopa augmentait encore d'avantage ce biais pour attendre les images retardées ainsi que cette activité cérébrale par rapport à la période sans traitement L-Dopa. Ces résultats identifient les sites anatomiques et l'effet de la LDopa sur le surcroît de motivation pour les images érotiques chez les patients hypersexuels et pourraient à terme servir à mieux cibler ces sites dans un but thérapeutique (Fig. 5).

De plus, une dévaluation par le délai serait un marqueur indépendant de l'évolution du jeu pathologique, qui pourrait en partie être en lien avec un déterminisme génétique.
Il existerait en effet une corrélation entre la dévaluation temporelle et le polymorphisme de gènes dû à plusieurs allèles codant pour certains récepteurs dopaminergiques (D2 et D4). Certaines différences génétiques entre individus pourraient d'autre part expliquer pourquoi le système dopaminergique répond différemment chez différentes personnes et expliquer en partie la relation entre réponse du système de récompense, variabilité génétique et la vulnérabilité à l'addiction, dont les addictions comportementales comme le jeu pathologique. Ainsi, nous avons montré que les polymorphismes de gènes impliqués dans la transmission dopaminergique, comme la Catéchol-0-méthyltransférase (COMT) et le transporteur de la dopamine (DAT) modulent l'activité du système de récompense [20] (Fig. 6).

La COMT est une enzyme qui joue un rôle critique dans le catabolisme de la dopamine libérée dans le cortex préfrontal, et le DAT joue également un rôle clé dans la disponibilité de la dopamine puisqu'il la recapture dans les terminaisons synaptiques, en particulier au niveau du striatum. Nous avons montré que moins il existe de dopamine au niveau synaptique (disposition génétique particulière) moins les sites de projection des neurones dopaminergiques (dans le cortex préfrontal latéral et dans le striatum ventral) sont engagés durant l'anticipation et 


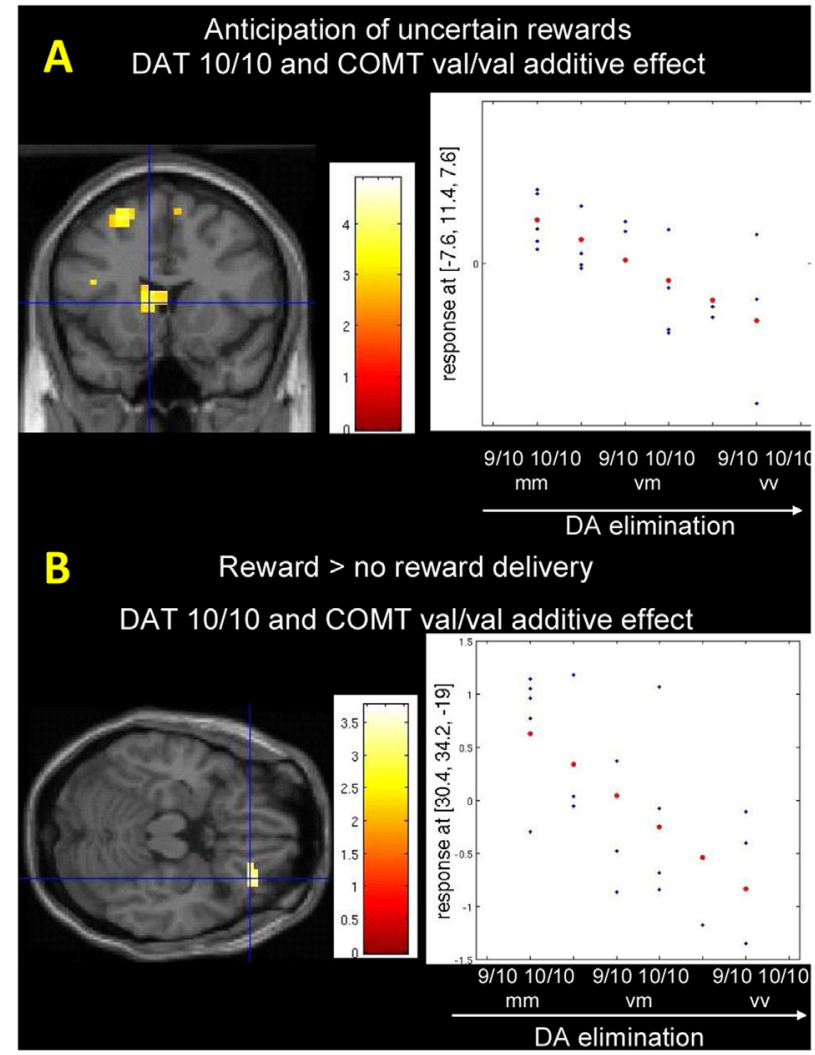

Figure 6 A. Effets additifs des génotypes COMT met/met et DAT1 9-répété montrant une activation plus forte du striatum ventral et du cortex orbitofrontal pendant l'anticipation et la réception d'une récompense d'incertitude maximale. À gauche : Cartes statistiques montrant les activations (réponses BOLD) en IRMf pendant l'anticipation et la réception d'une récompense d'incertitude maximale dans le striatum ventral superposées aux coupes IRM anatomiques. À droite : Corrélation négative entre le nombre d'allèles val (0_met/met, 1 _val/met, or 2_val/val) et DAT1 10- allèle répété (dosage (0_9/9 \& 9/10répété, 1_10/10-répété)) et l'activation du striatum ventral pendant l'anticipation d'une récompense d'incertitude maximale. B. Effets additifs des génotypes COMT met/met et DAT1 9-répété montrant une activation plus forte du cortex orbitofrontal pendant l'anticipation d'une récompense d'incertitude maximale. À gauche : Cartes statistiques montrant les activations (réponses BOLD) à la réception d'une récompense dans le cortex orbitofrontal superposées aux coupes IRM anatomiques. À droite : Corrélation négative entre le nombre d'allèles val (0_met/met, 1_val/met, or 2_val/val) et DAT1 10- allèle répété (dosage (0_9/9 \& 9/10-répété, 1_10/10-répété)) et l'activation du cortex orbitofrontal pendant l'anticipation d'une récompense d'incertitude maximale. La variation de la transmission dopaminergique associée au polymorphisme génétique met de la COMT et aux allèles 9-répétés VNTR DAT1 influence la réponse du système de récompense.

la réception de récompenses [20]. Ces réponses cérébrales pourraient contribuer aux différences interindividuelles dans les comportements de recherche de récompenses et dans les prédispositions à un grand nombre de troubles neuropsychiatriques comme l'addiction.

\section{Déséquilibre de la représentation de différentes récompenses chez les joueurs pathologiques}

Dans notre quotidien nous sommes régulièrement confrontés à des récompenses de nature diverses, parmi lesquelles nous devons bien souvent faire un choix. Il apparaît donc nécessaire que les entrées sensorielles et l'état motivationnel interagissent pour identifier les récompenses et comparer leurs valeurs respectives. Ce stade initial de traitement pourrait être codé par un réseau neuronal commun à différents types de récompenses. Dans une étude d'IRMf réalisée chez des sujets sains, notre équipe a testé l'existence à la fois de régions cérébrales communes et distinctes activées par des récompenses dont la nature diffère. Pour cela, on a comparé les réponses cérébrales pendant les phases d'anticipation et de réception de deux récompenses : les gains monétaires et les images érotiques [10,12,21-25] (Fig. 7).

Les résultats ont montré que la réception des récompenses fait appel à des régions cérébrales à la fois communes et distinctes. Concernant les régions communes, les analyses ont identifié plusieurs structures situées le long de la voie mésocorticolimbique, dont le cortex préfrontal ventromédian et le striatum ventral. Pour ce qui est des régions distinctes qui sont activées, une nette dissociation antéro-postérieure est présente au sein du cortex orbitofrontal latéral : alors que la région la plus antérieure est spécifiquement activée par la réception des gains monétaires, la région la plus postérieure est spécifiquement activée par la visualisation des images érotiques $[22,24,25]$. Ce résultat confirme l'hypothèse évolutionniste d'une dissociation entre récompenses primaires et secondaires dans le cortex orbitofrontal. Alors que les récompenses primaires, comme le sexe ou la nourriture, sont relativement archaïques et seraient donc représentées dans la partie postérieure du cortex orbitofrontal, c'est à dire la plus ancienne, les récompenses secondaires comme l'argent, apparues plus récemment dans l'évolution, seraient représentées dans des régions plus récentes au plan phylogénétique telles que le cortex orbitofrontal antérieur. Cette dissociation antéro-postérieure au sein du cortex orbitofrontal a récemment été confirmée par une méta-analyse sur plus de 80 études [25], montrant que cette dissociation reste aussi valide pour des récompenses primaires comme la nourriture. Ce paradigme a été utilisé par notre équipe pour tester les joueurs pathologiques. Une hypothèse concernant l'addiction consiste à penser qu'une suractivité du système de récompense pourrait conduire à un comportement excessif de recherche de plaisir. Cependant, les recherches en neuro-imagerie n'ont pas permis de valider cette hypothèse de façon claire chez les joueurs pathologiques. Alors qu'un certain nombre d'études conduites chez des joueurs pathologiques rapportent effectivement une hyperactivité du système de récompense en réaction aux gains monétaires, d'autres au contraire font état d'une hyposensibilité. Face à ce constat, nous avons proposé qu'en lieu et place d'une sensibilité exacerbée aux gains monétaires, les joueurs présentaient une sensibilité amoindrie aux autres types de récompenses, telles que la nourriture, le sexe, etc... ? Un tel déséquilibre de motivation entre plaisir monétaire et plaisir 


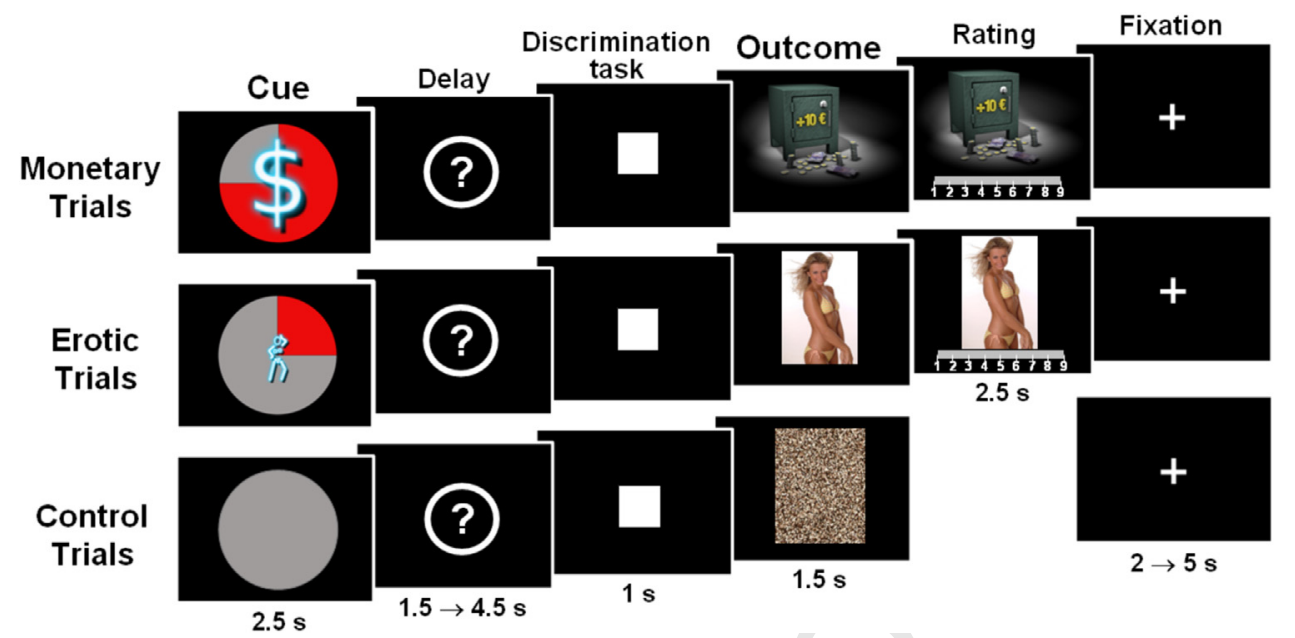

Figure 7 Protocole expérimental. Chaque essai du protocole, d'une durée variable de 8,5 à 17s, était découpé temporellement en trois phases : une phase d'anticipation de la récompense, suivie d'une tâche motrice de rapidité, puis d'une phase de réception de la récompense. La phase d'anticipation était matérialisée par un symbole annonçant les caractéristiques de la récompense à venir, à savoir sa probabilité, sa nature et sa magnitude. Les récompenses étaient soit de nature monétaire (en haut) ou érotique (milieu). Des essais «contrôles" (en bas) étaient associés à une probabilité nulle d'obtenir une récompense. Chaque essai se poursuivait par un délai d'attente, suivi d'une tâche motrice de type stimulus-réponse. L'obtention d'une récompense dans la phase finale de l'essai dépendait à la fois de la probabilité annoncée et de la réussite du sujet à la tâche motrice. En cas d'obtention le sujet devait évaluer son degré de satisfaction sur une échelle de 1 à 9 . Les essais de type monétaire et érotique étaient mélangés les uns aux autres. Le protocole comportait au total 171 essais, répartis sur 3 sessions de $12 \mathrm{~min}$, soit une durée totale de $35 \mathrm{~min}$ environ.

non-monétaire pourrait alors fournir une explication pour l'attrait particulier exercé par les jeux d'argent.

Pour tester cette hypothèse, des joueurs pathologiques et des sujets témoins ont été soumis à une IRMf lors d'un jeu fondé sur le même principe que celui des «machines à sous ", mais impliquant deux types de récompenses, à savoir des gains d'argent et des images érotiques [21]. La probabilité et l'intensité de ces récompenses étaient également manipulées. Les participants effectuaient une tâche de rapidité pour avoir une chance de gagner les sommes d'argent et de voir les images érotiques mises en jeu. Le résultat, qui est venu confirmer l'hypothèse émise, se décompose en trois parties [21].

Au niveau comportemental, alors que les sujets témoins montraient une motivation (mesurée par les temps de réponse) identique pour les gains monétaires et les images érotiques, les joueurs pathologiques avaient une motivation moindre pour les images érotiques comparativement aux gains monétaires [21]. Cette différence de comportement était visible au niveau cérébral : au cours de la phase d'anticipation des récompenses, il a été observé que le système de récompense des joueurs était nettement moins réactif aux images érotiques qu'aux gains monétaires, alors que le niveau d'activité cérébrale entre les images érotiques et les gains monétaires était similaire chez les sujets témoins [21] (Fig. 8).

Si cette différence de réactivité vis-à-vis des récompenses monétaires et non-monétaires est un réel « marqueur » du jeu pathologique, elle devrait être d'autant plus grande que les symptômes de jeu sont prononcés. C'est effectivement ce qui a été observé au niveau du striatum ventral [21]. De plus, l'activité au niveau du cortex orbitofrontal a montré des différences intéressantes entre joueurs pathologiques et sujets témoins. Alors que les sujets témoins montraient la même dissociation antéropostérieure au niveau du cortex orbitofrontal, que celle observée auparavant chez un autre groupe de sujets sains ; les joueurs pathologiques montraient eux, une activité similaire pour les récompenses monétaires et érotiques au niveau du cortex orbitofrontal latéral postérieur, normalement recruté uniquement par les récompenses primaires chez les sujets sains [10,12,21,22,24,25] (Fig. 9).

Tout se passe donc comme si les gains monétaires étaient éprouvés comme des récompenses primaires par les joueurs pathologiques. Cette possibilité est cohérente avec le fait que les joueurs semblent rechercher l'argent non pour ce qu'il permet d'acheter mais pour lui-même, comme s'il était intrinsèquement renforçant. Ces résultats mettent l'accent sur un dérèglement des processus motivationnels chez les joueurs pathologiques. D'un point de vue clinique, ils suggèrent une approche thérapeutique originale, focalisée sur la revalorisation des sources de plaisir autres que le jeu. Des recherches futures permettront de tester cette nouvelle hypothèse.

Nous avons par ailleurs montré qu'il existe des différences de morphologie au sein de l'organisation des sulci/gyri orbitofrontaux entre joueurs pathologiques et participants sains, et que la morphologie de cette région (antéro/postérieure) prédit le signal observé en IRMf pour des récompenses primaires vs secondaires [22,23]. Enfin, une méta-analyse de neuro-imagerie récente comparant l'addiction comportementale (jeu pathologique) et les addictions aux drogues (nicotine, cocaïne, cannabis, opioïdes, alcool) a montré une diminution de l'activation striatale pendant l'anticipation de la récompense chez les personnes ayant une dépendance par rapport à celles des groupes témoins [26]. Au moment de la délivrance de la récompense, les individus toxicomanes ont montré une 

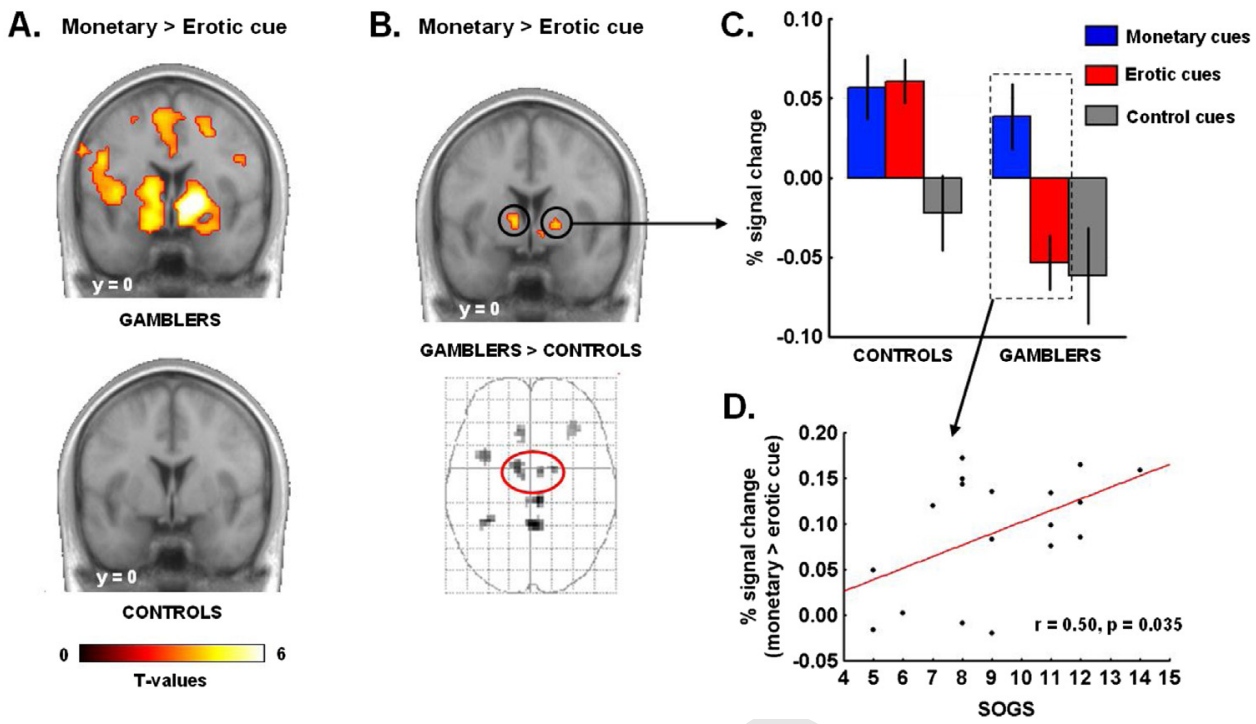

Figure 8 Hypoactivation du striatum ventral en réponse aux symboles prédictifs d'images érotiques chez les joueurs pathologiques. (A) Ces cartes montrent que le striatum ventral des joueurs (en haut) répond plus fortement aux symboles prédictifs de gains monétaires qu'aux symboles prédictifs d'images érotiques, alors que le striatum ventral des sujets sains (en bas) ne montre pas de différence. (B) Cette différence est significative lorsqu'on compare directement les deux groupes. (C) Ce graphe montre que la différence observée chez les joueurs est liée à une hypoactivation du striatum ventral en réponse aux symboles érotiques chez les joueurs pathologiques, et non à une hyperactivation en réponse aux symboles monétaires. (D) Enfin, ces résultats montrent que la différence de réactivité du striatum ventral aux symboles monétaires versus érotiques chez les joueurs pathologiques est corrélée positivement avec la sévérité des symptômes de jeu. Cette corrélation renforce l'idée que cette différence de réactivité est un véritable marqueur de l'addiction au jeu.

\section{Monetary reward > Control}

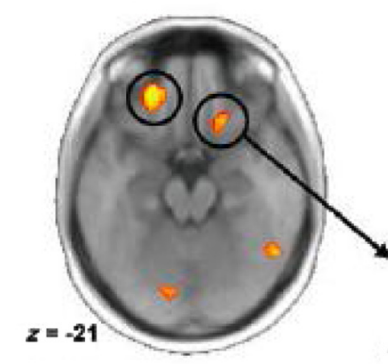

GAMBLERS > CONTROLS

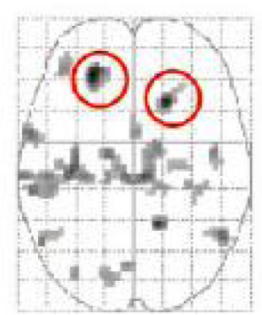

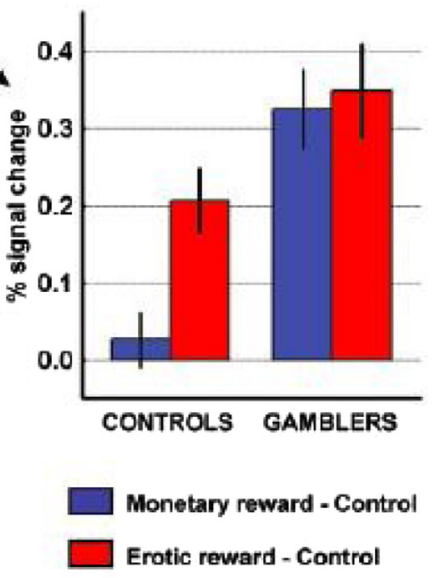

Figure 9 Activation du cortex orbitofrontal au moment de la réception des récompenses. Les résultats montrent que le cortex orbitofrontal postérieur latéral, normalement davantage recruté par les images érotiques (récompenses primaires) que par l'argent chez les sujets sains, est activé de la même façon par ces deux types de récompenses chez les joueurs pathologiques. Ces résultats suggèrent que l'argent pourrait être considéré comme une récompense primaire chez les joueurs pathologiques. activation accrue du striatum ventral, tandis que les individus dépendants du jeu ont montré une activation réduite du striatum dorsal par rapport aux témoins. Ces résultats concordent à la théorie d'un déficit ou déficience du système de récompense chez les joueurs pathologiques.

\section{Étude de l'impulsivité et du contrôle dans le domaine de la prise alimentaire}

Un autre domaine étudié par notre équipe, lié au comportement d'impulsivité concerne la prise alimentaire. Une question fondamentale est de comprendre comment notre cerveau régule-t-il la prise alimentaire lorsqu'on a faim. Nous avons récemment mis ont mis en évidence les réseaux cérébraux impliqués dans la régulation de l'appétit ainsi que le rôle des hormones contribuant à cette régulation [27].

La prise alimentaire est un comportement motivé par des nécessités internes d'ordre énergétique. Cependant, la prise alimentaire est aussi un comportement volontaire, qui peut être régulé consciemment. Un individu peut décider de se retenir de manger ou, à l'inverse, peut décider de continuer de manger même s'il perçoit la sensation de satiété. Par exemple, un individu suivant un régime peut décider de ne pas consommer de viennoiserie bien que la vue d'un pain au chocolat dans une vitrine de boulangerie, précédée par l'odeur de pain chaud émanant de cette dernière augmente son appétit. À l'inverse, ce même individu pourra se laisser tenter par cette même viennoiserie tout simplement parce qu'il veut se faire plaisir.

Pour tester si des régions cérébrales communes ou différentes sont engagées lors de la régulation à la hausse (se 


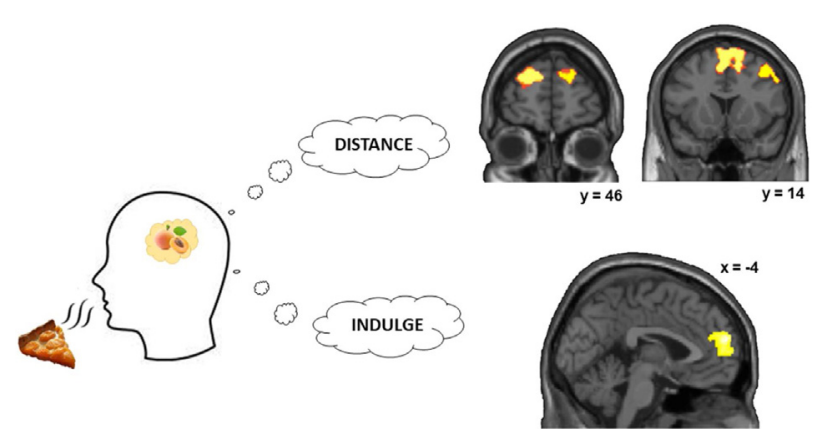

Figure 10 Lorsqu'on présente des stimuli de nourriture dans les modalités olfactives et visuelles dans le scanner, deux régions distinctes du cortex préfrontal sont engagées selon que l'individu se retient ou se fait plaisir lors de la perception des stimuli olfactifs et visuels. Le cortex préfrontal médian est impliqué dans la régulation à la hausse (se faire plaisir), et le taux de ghréline circulant augmente avec l'activité de cette région. La régulation de l'appétit à la baisse met en jeu le cortex préfrontal bilatéral médian et le cortex préfrontal dorsolatéral.

faire plaisir) et lors de la régulation à la baisse (se retenir de manger), nous avons proposé à un groupe de volontaires de participer à une expérience en IRM fonctionnelle. Les participants devaient donner le prix qu'ils étaient prêts à payer pour consommer un aliment dans différentes conditions : une condition 'Plaisir' où ils leur étaient demandé d'adopter des pensées qui augmenteraient leur désir de manger immédiatement les aliments présentés, une condition 'Retenue' où ils leur étaient demandé d'adopter des pensées qui diminuerait leur désir de manger immédiatement les aliments et une condition contrôle où aucune régulation n'était demandée. L'activité cérébrale des participants était enregistrée à l'aide d'un scanner IRMf (imagerie par résonance magnétique fonctionnelle) durant l'expérience. De plus, un olfactomètre compatible à l'IRMf permettait de délivrer une odeur de nourriture associée à chaque image tout en enregistrant des paramètres physiologiques respiratoires. À la fin de l'expérience, une prise de sang permettait la mesure des hormones homéostatiques que sont la ghréline et la leptine (Fig. 10).

Les résultats ont montré que les conditions de régulation modulent le prix que les individus sont prêts à payer pour consommer un aliment. Ces conditions affectent également les paramètres olfactifs mesurés lors de la présentation des odeurs : les participants respirent davantage de volume d'air lorsqu'ils décident de réguler positivement leur appétit. Au niveau cérébral, deux régions distinctes du cortex préfrontal sont engagées selon que l'individu se retient ou se fait plaisir lors de la perception des stimuli olfactifs et visuels. Le cortex préfrontal médian est impliqué dans la régulation à la hausse (se faire plaisir), et le taux de ghréline circulant augmente avec l'activité de cette région. La régulation à la baisse met en jeu le cortex préfrontal bilatéral médian et le cortex préfrontal dorsal médian. Ces résultats révèlent l'existence de différents systèmes cérébraux soutenant le processus de régulation de la prise alimentaire et soulignent l'importance du système olfactif et hormonal dans cette régulation. Ils revêtent une importance cruciale dans la compréhension des mécanismes de la prise alimentaire et pourraient s'avérer utile pour mieux comprendre

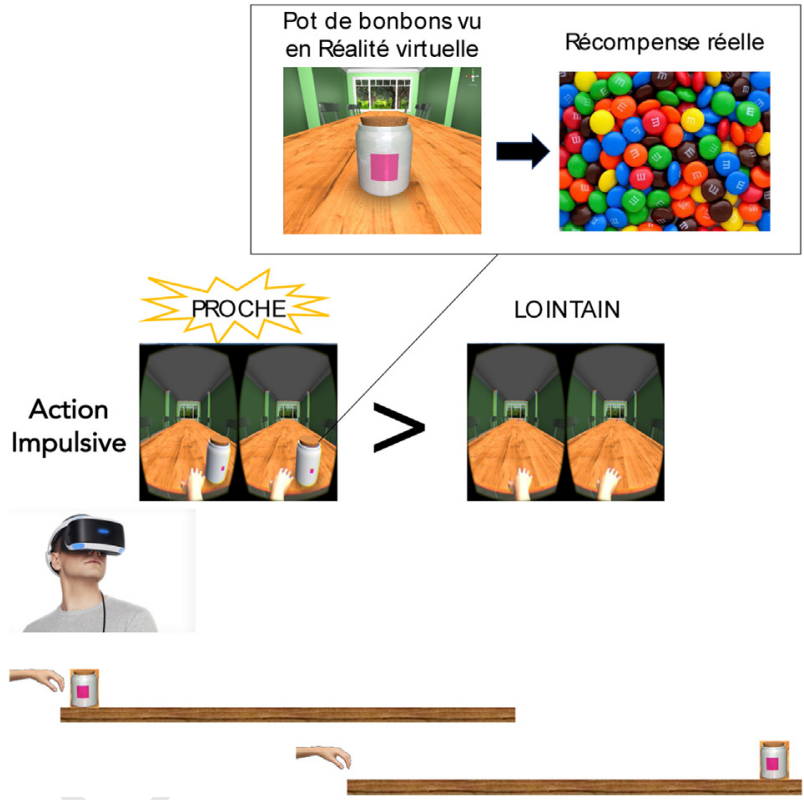

Figure 11 Lorsqu'on présente des stimuli de nourriture en réalité virtuelle $3 \mathrm{D}$, les participants ont davantage de difficulté à inhiber leurs réponses quand des symboles (ici carré rose sur le pot de M\&Ms) appétitifs de ne pas répondre (No-go) semblent proches comparé à lorsqu'ils sont éloignés.

les bases neurophysiologiques de troubles alimentaires (obésité, anorexie, ...) dans des conditions de prise alimentaire proches de la réalité (i.e. modalité olfactive et visuelle combinées).

Dans des environnements où nous sommes proches d'aliments à forte densité énergétique, comme aux caisses des supermarchés, nous avons tendance à consommer davantage. Cette observation confirme l'idée que le placement des aliments dans notre environnement peut avoir des effets délétères. La prévalence croissante de l'obésité pourrait être réduite par le changement du comportement grâce à des interventions simples, comme le placement plus éloigné de tels aliments à forte valeur énergétique. Comment la proximité physique aux aliments joue-t-elle dans la conduite d'actions impulsives pour la prise alimentaire?

Une question fondamentale est de savoir si cet effet de consommation impulsive d'aliments à proximité est dû à l'effort à fournir qui est moindre pour un aliment proche ou bien à la distance elle-même. On sait que lorsqu'on a à choisir entre deux récompenses (une faible et une plus importante), la valeur de l'option plus récompense diminue avec le coût de l'effort à fournir. Mais, comment distinguer ce coût de l'effort de celui lié à la distance à l'objet ? En utilisant la technologie de réalité virtuelle (VR), nous avons récemment réussi à isoler la propriété de proximité afin de mesurer son effet spécifique sur le comportement impulsif [28]. L'hypothèse générale était que la proximité spatiale aurait pour effet de nuire à la maîtrise de soi et donc d'augmenter le comportement impulsif. Les participants devaient répondre à des images tridimensionnelles représentant des récompenses alimentaires situées à proximité ou de façon plus lointaine (Fig. 11). Les analyses ont révélé que les participants étaient nettement moins capables pour arrêter les actions motrices lorsque les signaux gratifiants 
${ }_{79}$ Q4 [29-35]. étaient proches par rapport à loin. Des facteurs normalement associés à la proximité étaient contrôlés, comme l'effort-qui ne variait pas- ou la taille rétinienne des symboles présentés-qui ne différaient pas pour les conditions proches ou lointaines. Ces résultats montrent que la proximité joue un rôle distinctif dans la conduite d'actions impulsives pour des récompenses.

Ces résultats complètent des résultats récents de l'équipe ayant montré l'existence de différents systèmes cérébraux soutenant le processus de régulation de la prise alimentaire. Ils revêtent une importance cruciale dans la compréhension des mécanismes de la prise alimentaire et pourraient s'avérer utile pour mieux aider à développer des méthodes d'intervention (e.g. Nudge, architecture...) pour aider à réduire les troubles alimentaires dans des conditions de prise alimentaire réelles.

Enfin, un autre domaine d'étude passionnant est le mariage des connaissances en endocrinologie et en neuroimagerie pour l'étude des hormones dans les addictions comportementales et en neurosciences de la décision de façon plus générale. Nous renvoyons le lecteur à nos publications dans ce domaine.

\section{Conclusion}

Il existe des similitudes neurobiologiques, psychopathologiques et comportementales entre les addictions aux drogues et les addictions comportementales comme l'addiction au jeu. Au niveau du système de récompense, le jeu pathologique est caractérisé par une sensibilité striatale moindre aux récompenses non-monétaires. Les joueurs pathologiques présentent des biais cognitifs comme un biais d'optimisme vers les options plus risquées, quelles que soient les chances de gagner, et montrent également un dysfonctionnement du système de récompense et du système des valeurs.

Cet article a montré toute l'importance d'appliquer les méthodes de la neuroéconomie (Neurosciences de la décision) pour étudier les substrats cérébraux du traitement des récompenses et de la prise de décision dans l'addiction aux drogues, les perturbations de l'impulsivité (l'incapacité de résister à des tentations comme le jeu pathologique, ou l'hypersexualité), la maladie de Parkinson et l'anorexie [1].

\section{Déclaration de liens d'intérêts}

L'auteur déclare ne pas avoir de liens d'intérêts.

\section{Références non citées}

\section{Remerciements}

À Jean-Pierre Goullé et Jean-Pierre Olié pour la retranscription du texte.

\section{Références}

[1] Dreher JC, Tremblay L, editors. Decision Neuroscience. Academic Press, Elsevier; 2016. p. 422.

[2] Tversky A, Kahneman D. Judgment under Uncertainty: Heuristics and Biases. Science 1974;185:1124-31.

[3] Kahneman D, Tversky A. Prospect theory: An analysis of decision under risk. Econometrica 1979;47:263-91.

[4] Ligneul R, Sescousse G, Barbalat G, Domenech P, Dreher JC. Shifted risk preferences in pathological gambling. Psychol Med 2013;43:1059-68.

[5] Schultz W. Multiple functions of dopamine neurons. F1000 Biol Rep 2010;2:2.

[6] Metereau E, Dreher JC. The medial orbitofrontal cortex encodes a general unsigned value signal during anticipation of both appetitive and aversive events. Cortex 2015;63:42-54.

[7] Metereau E, Dreher JC. Cerebral correlates of salient prediction error for different rewards and punishments. Cerebral Cortex 2013;23:477-87.

[8] Dreher JC, Kohn P, Berman KF. Neural coding of distinct statistical properties of reward information in humans. Cerebral Cortex 2006;16:561-73.

[9] Dreher JC, Schmidt PJ, Kohn P, Furman D, Rubinow D, Berman KF. Menstrual cycle phase modulates reward-related neural function in women. Proceedings of the National Academy of Sciences 2007;104:2465-70.

[10] Sescousse G, Li Y, Dreher JC. A common currency for the computation of motivational values in the human striatum. Soc Cogn Affect Neurosci 2015;10:467-73.

[11] Domenech P, Redouté J, Koechlin E, Dreher JC. The neurocomputational architecture of value-based selection in the human brain. Cerebral Cortex 2018;28:585-601.

[12] Li Y, Vanni-Mercier G, Isnard J, Mauguière F, Dreher JC. The neural dynamics of reward value and risk coding in the human orbitofrontal cortex. Brain 2016;139:1295-309.

[13] Vanni-Mercier G, Mauguiere F, Isnard J, Dreher JC. The hippocampus codes the uncertainty of cue-outcome associations: an intracranial electrophysiological study in humans. Journal of Neuroscience 2009;29:5287-94.

[14] Dreher JC, Meyer-Lindenberg A, Kohn P, Berman KF. Agerelated changes in midbrain dopaminergic regulation of the human reward system. Proceedings of the National Academy of Sciences 2008;105:15106-11.

[15] Ojala K, Janssen L, Hashemi M, Timmer M, Geurts D, ter Huurne $\mathrm{N}$, et al. Dopaminergic drug effects on probability weighting during risky decision-making. eNeuro 2018;5(2.).

[16] Jupp B, Caprioli D, Saigal N, Reverte I, Shrestha S, Cumming P. Dopaminergic and GABA-ergic markers of impulsivity in rats: evidence for anatomical localisation in ventral striatum and prefrontal cortex. Eur J Neurosci 2013;37:1519-28.

[17] Miedl SF, Peters J, Büchel C. Altered neural reward representations in pathological gamblers revealed by delay and probability discounting. Arch Gen Psychiatry 2012;69: 177-86.

[18] Prévost C, Pessiglione M, Météreau E, Cléry-Melin ML, Dreher $J C$. Separate valuation subsystems for delay and effort decision costs. Journal of Neuroscience 2010;30:14080-90.

[19] Girard R, Obeso I, Thobois S, Park SA, Vidal T, Favre E, et al. Wait and you shall see: sexual delay discounting in hypersexual Parkinson's disease. Brain 2019;142:146-62.

[20] Dreher JC, Kohn P, Kolachana B, Weinberger DR, Berman KF. Variation in dopamine genes influences responsivity of the human reward system. Proc Natl Acad Sci U S A 2009;106:617-22.

[21] Sescousse G, Barbalat G, Domenech P, Dreher JC. Imbalance in the sensitivity to different types of rewards in pathological gambling. Brain 2013;136:2527-38. 
[22] Li Y, Sescousse G, Amiez C, Dreher JC. Local morphology predicts functional organization of experienced value signals in the human orbitofrontal cortex. J Neurosci 2015;35: 1648-58.

[23] Li Y, Wang Z, Boileau I, Dreher JC, Gelskov S, Genauck A, et al. Altered orbitofrontal sulcogyral patterns in gambling disorder: a multicenter study. Transl Psychiatry 2019;9:186.

[24] Sescousse G, Redouté J, Dreher JC. The architecture of reward value coding in the human orbitofrontal cortex. J Neurosci 2010;30:13095-104.

[25] Sescousse G, Caldú X, Segura B, Dreher JC. Processing of primary and secondary rewards: a quantitative meta-analysis and review of human functional neuroimaging studies. Neurosci Biobehav Rev 2013;37:681-96.

[26] Luijten $M$, Schellekens $A$, Kühn $S$, Machielse $M$, Sescousse G. Disruption of reward processing in addiction: An image-based meta-analysis of fMRI studies. JAMA Psychiatry 2017;74:387-98.

[27] Janet R, Fournel A, Fouillen M, Derrington E, Corgnet B, Bensafi $M$, et al. Cognitive regulation of food odor/image in the human brain. Neuroimage 2021 [In press].

[28] O'Connor D, Janet R, Guigon V, Belle A, Vincent BT, Bromberg U, et al. J.C. The proximity effect: rewards that are near increase impulsive action. iScience 2021 [In press].

[29] Li Y, Ramoz N, Derrington E, Dreher JC. Hormonal responses in gambling versus substance abuse: a review of human studies.
Progress in Neuro-Psychopharmacology and Biological Psychiatry 2020;100:109880.

[30] Li Y, Sescousse G, Dreher JC. Endogenous cortisol levels are associated with an imbalanced striatal sensitivity to monetary versus non-monetary cues in pathological gamblers. Frontiers in behavioral neuroscience 2014;8:83.

[31] Li Y, Météreau E, Obeso I, Butera L, Villeval MC, Dreher JC. Endogenous testosterone is associated with increased striatal response to audience effects during prosocial choices. Psychoneuroendocrinology 2020:104872.

[32] Li Y, Dreher JC. A review of the impact of hormone therapy on the prefrontal structure and function at menopause. Climateric 2021;11:1-10.

[33] Girard R, Météreau E, Thomas J, Pugeat M, Qu C, Dreher JC. Hormone therapy at early post-menopause increases cognitive control-related prefrontal activity. Scientific Reports 2017;7:44917.

[34] Dreher JC, Dunne S, Pazderska A, Frodl T, Nolan JJ, O’Doherty JP. Testosterone causes both prosocial and antisocial statusenhancing behaviors in human males. Proceedings of the National Academy of Sciences 2016;113:11633-8.

[35] Thomas J, Météreau E, Déchaud H, Pugeat M, Dreher JC. Hormonal treatment increases the response of the reward system at the menopause transition: A counterbalanced randomized placebo-controlled fMRI study. Psychoneuroendocrinology 2014;50:167-80. 